\title{
La relación entre los niveles de atención constituye un determinante clave de la salud*
}

\author{
The relationship among the various care levels is a key health \\ determinant
}

\author{
Patrick Van der Stuyft'; Pol De Vos"' \\ 'MD, PhD. Profesor Titular de Epidemiología. Instituto de Medicina Tropical. \\ Amberes, Bélgica. \\ "MD, MPH. Investigador en Salud Pública. Instituto de Medicina Tropical. Amberes, \\ Bélgica.
}

\section{RESUMEN}

Basándose en ejemplos concretos de sistemas de salud, se aportan elementos y argumentos al debate internacional sobre la importancia de los niveles de atención y de la relación óptima entre niveles. El primer nivel tiene tres funciones esenciales: ser el punto de entrada al sistema, facilitar y coordinar el itinerario de su paciente en el sistema, y asegurar la integración y la síntesis de la información de salud de cada paciente. Describiendo los diferentes obstáculos y problemas posibles, en primer lugar la lógica comercial que se ha ido introduciendo en muchos sistemas, los autores definen puntos de atención y proponen ajustes para asegurar el acceso universal, la equidad, la eficacia y eficiencia, y la sostenibilidad de un sistema de salud.

Palabras clave: Organización de servicios de salud, niveles de salud, funciones del primer nivel de atención, accesibilidad, calidad, eficacia, eficiencia, determinantes de la salud, salud pública.

\section{ABSTRACT}

On the basis of concrete examples of health systems, several elements and arguments are added to the international debate about the importance of health 
care levels and their optimal interrelationship. The first level has three main functions: being the entry point to the system, facilitating and coordinating the itinerary of a patient within the system, and guarantee the integration and summary of health information on each patient. By describing the various obstacles and possible problems, mainly the commercial logics that is being introduced in many systems, the authors defined points of attention and suggested adjustments to assure universal access, equity, efficiency and efficacy, and sustainability of a health care system.

Key words: Health service organization, health levels, functions of the primary care level, accessibility, quality, efficacy, efficiency, health determinants, public health.

\section{NTRODUCCI ÓN}

La declaración de Alma Ata estipula que la salud es un derecho fundamental cuya realización requiere una acción de muchos otros sectores sociales y económicos además del sector salud. ${ }^{1}$ El estado de salud de una población es el resultado de una acción multidisciplinaria con enfoque integral hacía sus determinantes políticos, sociales, económicos y ecológicos, incluyendo el desarrollo integral de servicios accesibles y de calidad. Sin duda, la contribución de los servicios de salud a la calidad de vida y la salud de una población es relativa, está plenamente comprobado que existen muchos otros determinantes, que son quizás más importantes. $^{2}$

No obstante, el desarrollo de servicios de salud es esencial para asegurar la promoción, la prevención, la curación y la rehabilitación de la salud y el bienestar. Además, el acceso a estos servicios es un derecho humano. Para asegurar este papel, los servicios deben organizarse de forma adecuada.

La tesis principal que se plantea en este artículo se puede resumir en que la definición y organización de los niveles de atención, junto a una óptima relación entre estos niveles, son condiciones necesarias para asegurar que el sector de la salud pueda asumir con calidad y eficacia su papel como uno de los determinantes importantes de la salud.

\section{LA DEFI NI CIÓN DE LOS NI VELES DE SALUD}

Clásicamente se distinguen tres niveles de atención. El primer nivel es el nivel más cercano a la población, o sea el nivel del primer contacto. En el segundo nivel se encuentran los hospitales de referencia, y el tercer nivel es el formado por hospitales de alta tecnología e institutos especializados.

Mientras que hay poca variabilidad en la conceptualización estructural y funcional del segundo y tercer nivel, existe una gran variedad de estructuras para el primer nivel de atención. Según el país este primer nivel puede ser formado por diferentes estructuras o formas organizativas: médicos de familia en consultorios individuales, equipo de médicos de familia ( $y$ a veces equipos multidisciplinarios con otros 
profesionales de la salud), centros de salud, policlínicos, o una combinación de estas variantes.

Pero lo que más difiere entre diferentes sistemas de atención en salud en el mundo, es el grado de definición explícita de los niveles de atención, de sus funciones, y de sus relaciones. Estas diferencias están relacionadas - pero no de manera absoluta o rígida- con el tipo de sistema y su modo de financiamiento. ${ }^{3,4}$

Algunos ejemplos típicos se muestran en la tabla 1.

El Servicio Nacional de Salud del Reino Unido tiene niveles de atención institucionalizados con un primer nivel bien definido en el cual los médicos de familia juegan un papel central. En este sistema los pacientes no tienen acceso directo al segundo nivel, pero siempre deben de entrar al sistema de salud a través de su médico de familia (o el servicio de urgencias). ${ }^{5}$

En el sistema de seguro social de Bélgica el primer nivel tiene un papel reconocido, y aquí también el médico de familia juega un rol preponderante. Pero contrario a lo que ocurre en el Reino Unido, este primer nivel no está institucionalizado. En principio, cada paciente tiene acceso directo al especialista del segundo o tercer nivel. El sistema tiene además un importante grado de comercialización: el proveedor es un médico privado, y el paciente paga la consulta según tarifas del seguro social, quien reembolsa aproximadamente $2 / 3$ del costo al paciente. Esta comercialización implica que existe una competición entre médicos y servicios tanto dentro de cada nivel como entre los niveles, lo cual tiende a romper los niveles. ${ }^{6}$ En contra de esta tendencia existe un movimiento de médicos progresistas que intenta fortalecer la nivelización, y aboga para reforzar la inscripción de pacientes con un médico de familia fijo. Además se propone un sistema con un pago mensual (por parte del seguro social) por cada paciente inscrito, mientras que la atención es gratuita para el paciente. ${ }^{7,8}$

En Estados Unidos el sistema de salud se rige por la atención gerenciada, lo que implica que las grandes corporaciones aseguradoras compiten por afiliados en un "mercado de salud." A nivel de los servicios se observa la casi inexistencia del primer nivel e incluso de una definición de niveles. La lógica comercial rige el desarrollo de las instituciones, implicando que las compañías aseguradoras traten de contratar la mayor cantidad de personas, y a la vez limitan sus gastos de servicios de salud en caso de enfermedad. Al final de cuentas, la lógica financieraque no busca la eficacia pero la rentabilidad- tiende a limitar el acceso a los servicios de salud. ${ }^{10-12}$

En América Latina, la mayoría de los sistemas de salud están conformados por un sector privado, un seguro social para las personas con un trabajo formal, y un servicio público - muchas veces subfinanciado- para los pobres, quienes en muchas ocasiones forman la mayoría. Estos sistemas fragmentados tienen una nivelización variada, ya que mezclan elementos de los tres ejemplos elaborados anteriormente. $^{13}$

Lo que puede sorprender -o quizás no tanto en un segundo análisis- es la relación entre el grado de definición de niveles, los gastos y los resultados de los diferentes sistemas. Efectivamente, aunque existen importantes factores de confusión que no se incluyen en este análisis, se observa una relación inversa entre el grado de definición y de organización de los niveles (definiendo el primer nivel como punto de entrada del sistema y limitando la entrada directa a los otros niveles) y los gastos totales en salud, y una relación positiva entre el grado de nivelización efectiva y los resultados de salud (tabla 2 ). 
En resumen, una definición apropiada de los niveles, además de su apropiado, funcionamiento tiende a mejorar los resultados y a reducir los gastos, lo cual resulta en un sistema con mayor costo-eficacia. Además, como se analiza abajo, el funcionamiento adecuado del primer nivel de atención es un elemento clave para alcanzar estos resultados.

\section{LAS FUNCI ONES DE LOS NI VELES}

Generalmente se acepta que el segundo y tercer nivel deben complementar al primero, en el sentido de que deben hacer lo que el primer nivel no puede hacer por razones de eficacia y eficiencia, entre ellos, los diagnósticos y tratamientos especializados (aunque la tecnicidad de los niveles puede variar entre países).

Para el primer nivel, la tecnicidad es un factor importante pero no preponderante. Aquí, el elemento esencial es la buena organización de los servicios, y solamente se debe prever la tecnología necesaria para poder cumplir las tres funciones esenciales del primer nivel: 1 . Ser punto de entrada al sistema, 2. Facilitar y coordinar el flujo del paciente dentro del sistema, y 3 . Asegurar la integración y hacer la síntesis de los diferentes problemas, diagnósticos y tratamientos para el manejo adecuado del paciente.

La función de punto de entrada es esencial para la eficiencia del sistema, ya que disminuye la (sobre)demanda en los otros niveles. Pero obviamente eficiencia no significa que hay que convertir el punto de entrada en una barrera. Este riesgo de anteponer el control de gastos a las necesidades es un riesgo que amenaza en el sistema en el Reino Unido, donde se convirtió al médico de familia en "tenedor de fondos" (fund holder) quien "compra" los servicios necesarios para sus pacientes en los otros niveles de salud. ${ }^{14-16}$ En este sistema, los médicos de familia corren el riesgo de ser penalizados si gastan "demasiado". En el extremo, en sistemas de atención gerenciada donde más bien la rentabilidad es el objetivo, los aseguradores retoman el control de los profesionales (del primer u otro nivel) y limitan activamente el acceso a los servicios. ${ }^{17,18}$ Para ser funcional, el primer nivel también necesita tener la competencia suficiente y la tecnicidad adecuada para poder resolver gran parte de los problemas de salud comunes. Si no, arriesga de limitar su función a una ventanilla de entrada innecesaria e inútil hacia los otros niveles, lo cual solamente retrasaría los procesos y las soluciones.

La facilitación y coordinación es la segunda función clave del primer nivel (o de profesionales específicos en este nivel). Consiste en elaborar y negociar el itinerario en el sistema con el mismo paciente y con los otros profesionales de la salud con especialización diferente. ${ }^{19,20}$ Además, incluye asistir al paciente en este itinerario, asegurando su comprensión y adhesión a los exámenes y tratamientos propuestos, y garantizar una referencia y contrarreferencia adecuada. ${ }^{21}$ Así se asegura la continuidad de la atención durante un episodio de enfermedad determinada. En los países con sistemas eficientes, es el médico de familia quien se encarga de esta función del primer nivel. ${ }^{22}$

Finalmente, la tercera función de integración y síntesis asegura la eficacia. Sólo un médico de familia puede cumplir con ella, porque es el profesional que está suficientemente cerca del paciente (su paciente), de su entorno familiar, y del ecosistema directo donde vive. ${ }^{23}$ La tecnología esencial para lograr esta integración es el expediente personal y familiar sistemático y de calidad. Con la integración y síntesis se asegura la continuidad (también a largo plazo) y una atención integral. ${ }^{24,25}$ Una continuidad de atención adecuada necesita un ambiente de atención estable, se basa en una buena comunicación entre el médico y el paciente, 
y tiene el propósito de realmente obtener una mejoría de la salud y/o del bienestar del paciente. ${ }^{26}$

Estas tres funciones del primer nivel mencionadas son importantes para una atención de salud eficiente, eficaz y de calidad a todas las edades. Pero son particularmente estratégicas para el desarrollo de una respuesta adecuada al problema del envejecimiento de la población, que es actualmente una prioridad y un desafío para la salud pública en muchos países. El seguimiento de las enfermedades crónicas y el apoyo médico-social adecuado en función del ambiente social y familiar del paciente hace imprescindible la existencia de un médico que conozca a su paciente, que pueda acompañarlo en el manejo integral de su salud y que asegure la continuidad de atención a largo plazo, incluso en la última fase de la vida. ${ }^{27}$ Aquí el médico de familia demuestra su papel clave para asegurar la eficiencia y la eficacia en términos de salud y calidad de vida.

\section{HACI A LA EDUCACIÓN DE LOS NI VELES}

Probablemente no existe ningún país en el mundo donde la definición de los niveles y su interacción funciona a la maravilla, los posibles obstáculos son muchos. A nivel del concepto mismo de un sistema de salud, la amenaza más importante a nivel mundial es el avance de la lógica comercial que estimula la competición entre profesionales de diferentes niveles. También la falta de una definición adecuada de la población de responsabilidad de cada unidad del primer nivel puede causar dificultades. Además, las pléyadas de programas no integrados, que podrían estar brindándose a través de estructuras paralelas, resulta en una segmentación de la atención en el primer nivel y dificulta una atención integral. Y por fin, la inestabilidad del personal médico y paramédico es una plaga que en muchos países afecta sobre todo el personal del primer nivel, donde precisamente la estabilidad es lo más importante para construir una relación a largo plazo y de confianza entre el profesional de salud y la población. ${ }^{25,28,29}$

A nivel de recursos cabe mencionar la necesidad de una formación más adecuada de los recursos humanos, entro otros de los médicos de familia y de los especialistas, para que no solamente sean buenos técnicos y estén empapados de la función humanista del profesional de la salud, pero también comprendan la importancia de las funciones de los niveles en la lógica del sistema y el papel del primer nivel.

Otra limitante es a menudo la falta de poder resolutivo, tanto en países en desarrollo como en países industrializados, sobre todo a nivel del médico de familia. Sin embargo, para adecuar este nivel resolutivo no necesariamente se debe prever aquí toda la tecnología que los recursos financieros del sector salud permitieran, y mucho menos toda la tecnología disponible y comercialmente recomendada. Al contrario, demasiada tecnicidad va en contra de las funciones esenciales del médico de familia, puerta de entrada e integración de los problemas de salud del paciente. Finalmente, los profesionales del primer nivel deben de tener una carga de trabajo aceptable para poder ejercer sus funciones correctamente. Aquí se imponen ajustes a veces en el índice recursos/población y en la división de tareas dentro del equipo de salud, incluyendo las tareas administrativas.

Estos obstáculos y problemas pueden estar presentes tanto a nivel de organización del sistema como a nivel de recursos, pero deben resolverse. Niveles de salud adecuados y funcionales son una condición necesaria para garantizar el acceso universal, la equidad, la eficacia y eficiencia, y la sostenibilidad de un sistema de salud. 


\section{REFERENCI AS BI BLI OGRÁFICAS}

1. Alma Ata Declaration. International Conference on Primary Health Care. [serie en Internet]. [citado 15 de Abr 2008]. Disponible en:

http://www. who.int/hpr/NPH/docs/declaration_almaata.pdf [Alma-Ata, USSR, 6-12 September 1978].

2. Álvarez-Pérez AG, García Fariñas A, Bonet Gorbea M. Pautas conceptuales y metodológicas para explicar los determinantes de los niveles de salud en Cuba. Rev Cubana Salud Pública [serie en Internet]. 2007 [citado 15 Abr 2008]; 33. Disponible en: http://scielo.sld.cu/scielo. php?script $=$ sci arttext\&pid=S0864-

$\underline{34662007000200013 \& \operatorname{lng}=e s \& n r m=i s o \& t \mid n g=e s}$

3. Roemer MI. National Health Systems of the World. V1. New York: Oxford University Press; 1991.

4. OECD. OECD Health Data 2001. Paris: CREDES; 2001.

5. Chen GJ , Feldman SR. Economic apects of health care systems. Advantage and disadvantage of incentives in different systems. Dermatologic Clin.

2000; 18(2): 211-4.

6. Foets M, Berghmans F, Janssens L. The Primary Health Care Project in Belgium: a survey on the utilization of health services. Soc Sci Med. 1985;20(3):181-90.

7. Nonneman W, Van Doorslaer E. The role of the sickness funds in the Belgian health care market. Soc Sci Med. 1994;39(10):1483-95.

8. Arnaert A, Heuvel BV, Windey T. Health and social care policy for the elderly in Belgium. Geriatr Nurs. 2005;26(6):366-71.

9. McLean B. The big money in Medicaid. A boom in HMOs for the neediest leads to litigation, controversy-and lots of profits. Fortune. 2007; 155(12):97-102.

10. Cunningham R. Perspectives. Medicaid HMOs: going gets tougher in new environment. Med Health. 1998; 52(2): suppl 1-4.

11. Rizzo J A, Xie Y. Managed care, consumerism, preventive medicine: does a causal connection exist? Manag Care Interface. 2006; 19(7):46-50.

12. Benko LB. It's a seller's market. Insurers turn to managed care. Mod Healthc. 2005; 35(6): 7-15.

13. De Vos P, De Ceukelaire W, Van der Stuyft P. Colombia and Cuba: contrasting models in Latin-American health sector reform. Trop Med Int Health.

2006; 11(10): 1604-12.

14. Dusheiko M, Gravelle H, Yu N, Campbell S. The impact of budgets for gatekeeping physicians on patient satisfaction: evidence from fundholding. J Health Econ. 2007; 26(4): 742-62. 
15. Milne RG, Torsney B. Financial incentives, competition and a two tier service: lessons from the UK National Health Service internal market. Health Policy. 2003; 64(1): 1-12.

16. Propper C, Wilson D, Söderlund N. The effects of regulation and competition in the NHS internal market: the case of general practice fundholder prices. J Health Econ.1998; (6):645-73.

17. Lesser CS, Ginsburg PB. Health care cost and access problems intensify: initial findings from HSC's recent site visits. Issue Brief Cent Stud Health Syst Change. 2003; 63: 1-6.

18. Draper DA, Ginsburg PB. Health care cost and access challenges persist: initial findings from HSC's 2007 site visits. Issue Brief Cent Stud Health Syst Change. 2007; 114: 1-6.

19. Tjerbo T, Kjekshus L. Coordinating health care: lessons from Norway. Int J Integr Care. 2005; 5-28.

20. Segal L. The importance of patient empowerment in health system reform. Health Policy. 1998; 44(1):31-44.

21. Rosemann T, Wensing M, Rueter G, Szecsenyi J. Referrals from general practice to consultants in Germany: if the GP is the initiator, patients' experiences are more positive. BMC Health Serv Res. 2006; 19:5-6.

22. Guthrie B, Wyke S. Personal continuity and access in UK general practice: a qualitative study of general practitioners' and patients' perceptions of when and how they matter. BMC Fam Pract. 2006; 7: 11.

23. Kearley KE, Freeman GK, Heath A. An exploration of the value of the personal doctor-patient relationship in general practice. Br J Gen Pract. 2001;51(470): 7128.

24. Schers H, Webster S, van den Hoogen H, Avery A, Grol R, van den Bosch W. Continuity of care in general practice: a survey of patients' views. Br J Gen Pract. 2002; 52(479) : 459-62.

25. Stokes T, Tarrant C, Mainous AG, Schers H, Freeman G, Baker R. Continuity of care: is the personal doctor still important? A survey of general practitioners and family physicians in England and Wales, the United States, and The Netherlands. Ann Fam Med. 2005;3(4): 353-9.

26. Sturmberg J. Continuity of care: towards a definition based on experiences of practising GPs. Fam Pract. 2000; 17(1): 16-20.

27. Michiels E, Deschepper R, Van Der Kelen G, Bernheim JL, Mortier F, Vander Stichele R, Deliens L. The role of general practitioners in continuity of care at the end of life: a qualitative study of terminally-ill patients and their next of kin. Palliat Med. 2007;21(5):409-15.

28. Guthrie B. Continuity in UK general practice: a multilevel model of patient, doctor and practice factors associated with patients seeing their usual doctor. Fam Pract. 2002; 19(5): 496-9. 
29. Tarrant C, Windridge K, Boulton M, Baker R, Freeman G. How important is personal care in general practice? BMJ. 2003; 326(7402): 1310.

Recibido: 15 de mayo de 2008.

Aprobado: 4 de junio de 2008.

Pol De Vos. Instituto de Medicina Tropical, Unidad de Epidemiología y Control de Enfermedades, Departamento de Salud Pública. Nationalestraat 155, 2000 Antwerp, Bélgica.

Telef.:

003232476285 .E-mail: pdevos@itg.be

* Basado en una presentación oral en el Congreso de Higiene y Epidemiología 2007, La Habana, 5 al 9 de noviembre 2007.

Tabla 1. Definición de niveles de atención en diferentes sistemas

\begin{tabular}{|c|c|c|}
\hline Definición de los niveles & Tipo de sistema & Ejemplos \\
\hline$* * *$ & Servicio Nacional de Salud & $\begin{array}{l}\text { Reino Unido } \\
\text { - Primer nivel institucionalizado } \\
\text { - Rol central del médico de familia } \\
\text { - No hay acceso directo al segundo nivel }\end{array}$ \\
\hline$+/-$ & Seguro Social & $\begin{array}{l}\text { Bélgica } \\
\text { - Primer nivel tiene papel reconocido } \\
\text { - Rol central del médico de familia } \\
\text { - No institucionalizado: Lógica comercial rompe } \\
\text { niveles }\end{array}$ \\
\hline- & Seguros privados & $\begin{array}{l}\text { Estados Unidos } \\
\text { - Primer nivel casi inexistente } \\
\text { - Servicios: anarquía comercial (mercado de salud) } \\
\text { - Presión de seguros para limitar acceso }\end{array}$ \\
\hline Variado & Mixto & América Latina \\
\hline
\end{tabular}


Tabla 2. Grado de definición de niveles, gastos y resultados

\begin{tabular}{|c|c|c|c|c|c|c|}
\hline $\begin{array}{l}\text { Definición } \\
\text { de niveles }\end{array}$ & Ejemplos & $\begin{array}{c}\text { Gasto total } \\
\text { en salud } \\
\text { ppcusD/ } \\
\text { persona } \\
\text { (1999) }\end{array}$ & $\begin{array}{l}\text { Gasto } \\
\text { público en } \\
\text { salud } \\
\% \text { del gasto } \\
\text { total (1998) }\end{array}$ & $\begin{array}{l}\text { Esperanza } \\
\text { de vida } \\
\text { (1995- } \\
2000 \text { ) }\end{array}$ & $\begin{array}{l}\text { Probabilidad } \\
\text { (al nacer) de } \\
\text { morir< } 60 \text { años } \\
\text { (\%) }\end{array}$ & $\begin{array}{c}\text { AVP } \\
\text { antes< } 70 \\
\text { años por } \\
100000\end{array}$ \\
\hline$* * *$ & $\begin{array}{l}\text { Reino } \\
\text { Unido }\end{array}$ & 1532 & 83,3 & 77,2 & 9,9 & 4061 \\
\hline$+/-$ & Bélgica & 2172 & 71,2 & 77,9 & 10,5 & 4537 \\
\hline- & $\begin{array}{l}\text { Estados } \\
\text { Unidos }\end{array}$ & 4180 & 44,8 & 76,5 & 12,8 & 5894 \\
\hline
\end{tabular}

ppcUSD/persona: USD paridad de poder de compra, AVP: años de vida perdidos (antes de los 70 años). 